\title{
Exploring and Overcoming the Challenges Primary Care Practices Face with Care Management of High-Risk Patients in CPC+: a Mixed-Methods Study
}

\author{
Tricia Collins Higgins, Ph.D., M.P.H. ${ }^{7}$ (D) Ann S. O'Malley, M.D., M.P.H. ${ }^{7}$, and \\ Rosalind E. Keith, Ph.D., M.H.S.A. ${ }^{2}$ \\ ${ }^{1}$ Mathematica, Washington, DC, USA; ${ }^{2}$ Mathematica, Princeton, NJ, USA.
}

\begin{abstract}
BACKGROUND: Longitudinal care management (LCM) for high-risk patients is a cornerstone of primary care models aiming to improve quality and reduce costs.

OBJECTIVE: Describe the extent to which LCM was implemented in the second year of Comprehensive Primary Care Plus (CPC+), and barriers to and facilitators of implementation.
\end{abstract}

DESIGN: Mixed-methods.

PARTICIPANTS: Quantitative: 2715 practices participating in $\mathrm{CPC}+$ in 2018. Qualitative: Interviews with practitioners and staff in 23 representative $\mathrm{CPC}+$ practices.

MAIN MEASURES: Across all CPC+ practices, we report median percentages of empaneled patients placed in the highest-risk tiers and, of those, the median percentage receiving LCM. Across $23 \mathrm{CPC}+$ practices, we report qualitative findings on LCM implementation.

KEY RESULTS: While practices reported benefits of LCM, a small proportion of patients received LCM. Practices placed $2.4 \%$ (median) of patients in the highest-risk tier; of these, 30\% (median) received LCM. Practices placed $10 \%$ (median) of patients in the second-highest-risk tier; of these, $7 \%$ (median) received LCM. Interviews revealed LCM uptake across tiers was low because of insufficient care manager staffing. Other challenges included lack of practitioner buy-in to using risk stratification to identify high-risk patients, patients' reluctance to engage in LCM or change behaviors, and limited health information technology functionality for developing, maintaining, and accessing high-risk patients' care plans. Facilitators included embedding care managers within practices and electronic health record functionalities that support LCM. CONCLUSIONS: Despite substantial financial and other supports, and practices' perceived benefits of LCM, insufficient care manager staffing and other barriers have limited its potential in CPC+ to date. To expand LCM's reach, practices need additional care managers, training to overcome barriers to patient engagement, better identification of patients who might benefit from LCM, improved information technology tools for risk stratification and care plans, and more practitioner buy-in to risk stratification.

KEY WORDS: care management; risk stratification; high-risk patients; care plans; primary health care.

Prior Presentations None.

Received August 17, 2020

Accepted December 20, 2020

Published online January 26, 2021
J Gen Intern Med 36(10):3008-14 DOI: $10.1007 / \mathrm{s} 11606-020-06528-0$

(c) Society of General Internal Medicine 2021

\section{INTRODUCTION}

To improve quality and reduce costs, primary care redesign initiatives often include risk stratification to identify patients with different levels of need and targeted, proactive, relationship-based longitudinal care management (LCM) for patients with the highest needs. The Comprehensive Primary Care Plus (CPC+) model - a 5-year, multipayer model sponsored by the Centers for Medicare \& Medicaid Services (CMS) - is one major example of such initiatives. ${ }^{1,2} \mathrm{CPC}+$ aims to strengthen primary care by developing five primary care functions: access and continuity of care, care management, comprehensiveness and coordination of care, patient and caregiver engagement, and planned care and population health. These changes are hypothesized to improve health, lower costs, and enhance patients' and providers' experience. CPC+ practices in this study joined one of two tracks in 2017: Track 1 and 2 practices had the same core set of requirements; in addition, Track 2 practices had extra requirements to better serve patients with complex needs. Due to the additional Track 2 requirements, these practices received more financial support than Track 1 practices. At the start of $\mathrm{CPC}+$, CMS collaborated with more than 70 commercial and state health insurance plans in 14 regions across the USA to provide more than 2900 primary care practices with financial support. This financial support included enhanced payments (in addition to usual payments for services) for participating in $\mathrm{CPC}+$ and for improving performance on cost, utilization, or quality measures; it also provided payments that move away from volume-based incentives for Track 2 practices. In 2018, the second year of the model, practices received enhanced payments representing a median of $10 \%$ of Track 1 practices' total revenue and $15 \%$ of Track 2 practices' total revenue. Median enhanced payments calculated per primary care practitioner were around $\$ 43,000$ for Track 1 practices and $\$ 66,000$ for Track 2 practices. CPC+ also provided regular data feedback, learning support, and health information technology support. 
As part of care management, all $\mathrm{CPC}+$ practices had to implement a two-step risk stratification process to identify patients for LCM: (1) use an algorithm of practices' choosing (such as the American Academy of Family Physicians risk stratification algorithm, an EHR-based tool, or an algorithm developed by the practice) based on defined diagnosis, claims, or other electronic data to assign a risk score to all empaneled patients and (2) use care team members' knowledge of the patient to adjust the risk score. Based on the results of the risk stratification process, all $\mathrm{CPC}+$ practices were required to provide targeted, proactive relationship-based LCM to all patients identified as at increased risk and likely to benefit from intensive care management, including patients with some combination of multiple comorbidities, complex treatment regimens, frailty and functional impairment, behavioral and social risks, and serious mental illness. In its guidance to practices, CMS suggested a typical population distribution has about $3-5 \%$ of the patient population at high risk, with no more than $10 \%$ of the population receiving care management services. ${ }^{3}$

LCM services include educating patients to manage their chronic conditions, working with patients during and between primary care visits, and monitoring care transitions such as after a hospitalization. Although CMS does not require care managers, it encourages practices to use on-site, non-physician, practice-based, or integrated shared care managers to provide LCM. Track 2 practices (but not Track 1 practices) were also required to use care plans to document and track patients' needs and how the practice addresses them. ${ }^{3}$

Potential benefits of LCM include improved quality of care and improved patient experiences. ${ }^{4-6}$ Evidence of LCM's effects on reducing hospitalizations, emergency department use, and cost savings is mixed. A recent multiyear study of a Pioneer accountable care organization found that participation in a care management program was associated with substantial reductions in hospitalization rates, emergency department visits, and Medicare spending. ${ }^{7}$ Another recent study of accountable care organization patients in the Medicare Shared Savings Program found significant reductions in total Medicare fee-for-service spending for these patients but proportionately smaller reductions in hospitalizations - and some increases in hospitalizations - for ambulatory care-sensitive conditions. $^{8}$

Despite the potential benefits of LCM, research indicates that implementing risk stratification and LCM is challenging for primary care practices. Risk stratification is relatively new to primary care, and methods to identify high-risk patients are imperfect. Practices' trust in risk scores could be influenced by the availability of patient information to assign risk (including factors such as social support, patient motivation, and nonmedical life changes) and care team members' buy-in to the value and accuracy of risk stratification. ${ }^{9,}{ }^{10}$ Even when highrisk patients are appropriately identified, barriers to implementing LCM include the fee-for-service payment system, which does not typically provide incentives to implement
LCM; resistance to change within primary care practices; lack of staff, resources, and knowledge necessary to integrate LCM into primary care practices; and inadequate health information technology to support LCM. ${ }^{11}$

Given the growing number of primary care practices attempting to implement risk stratification and LCM, CPC+ provides an opportunity to understand how practices in a model with robust financial and learning supports approach this work. This mixed-methods study describes the extent to which LCM reached high-risk patients in the second year of $\mathrm{CPC}+$, discusses why implementing LCM was challenging, and identifies factors that helped practices overcome challenges.

\section{METHODS}

The New England Institutional Review Board (IRB) granted this study an IRB exemption on the basis of the federal common rule (section 45 CFR 46.101[b][5]), because the purpose of the study was to evaluate a public benefit program.

\section{Quantitative Analysis}

Data. After each quarter of $\mathrm{CPC}+$, CMS requires all participating practices to answer a series of questions about how they perform activities related to various care delivery requirements. These are $\mathrm{CPC}+$ requirements related to each of the five primary care functional areas, including care management. ${ }^{12}$ All $\mathrm{CPC}+$ practices must answer the same care delivery requirement questions. These data enable CMS to track the proportion of practices that report implementing various $\mathrm{CPC}+$ activities; these data are self-reported, however, and provide less information on the intensity with which practices implement these activities. In this study, we report on care delivery requirement data that $\mathrm{CPC}+$ practices submitted to CMS in quarter four $2018(n=2715)$, at the end of their second year of participation in CPC+.

Analytic Approach. Across all $2715 \mathrm{CPC}+$ practices, we calculated the median proportion of empaneled patients that practices reported assigning to each of their risk tiers. In year 2 of $\mathrm{CPC}+$, all practices were required to maintain empanelment of at least $95 \%$ of their patient population to a practitioner. For each risk tier grouping, we calculated the median proportion of patients that practices reported were receiving LCM.

\section{Qualitative Analysis}

Data. In 2019, Mathematica conducted semistructured telephone interviews with a representative sample of $\mathrm{CPC}+$ practices that started participating in 2017. In choosing practices for the in-depth LCM study, we prioritized approximating a match between the percentage of practices in the 
LCM sample to the percentage of $\mathrm{CPC}+$ practices overall in terms of CPC+ track, participation in the Medicare Shared Savings Program for accountable care organizations, and ownership status (independent or owned by a system, hospital, or large group practice). Our LCM in-depth sample was also fairly similar to $\mathrm{CPC}+$ practices overall in terms of practice size (measured by number of primary care practitioners - physicians, nurse practitioners, physician assistants - at the practice site) [Table 1]. Researchers collected qualitative data on LCM, as well as topics outside the scope of this study (for example, other $\mathrm{CPC}+$ primary care functions, payment and learning supports, and use of specialists and teamwork). In order to cover all of these topics in-depth across the 59 practices in the qualitative study without placing undue burden on practices, we used a modular approach to data collection in which we randomly assigned two to three interview topics to each of the 59 practices. In doing so, 23 of the 59 practices received in-depth questions related to LCM. Therefore, our qualitative results stem from analysis of in-depth LCM interviews in these 23 practices. In each practice, one trained researcher interviewed three to four respondents, including at least one physician and one care manager (usually a registered nurse or nurse practitioner) for 30-60 min each. In some practices, we interviewed practice managers and medical assistants who were involved with risk stratification and LCM. In system-owned practices, we also interviewed system-level staff, such as CPC+ coordinators or centralized care managers. We asked questions to capture what practices did to implement risk stratification and LCM and the challenges and facilitators to implementation. We recorded and transcribed all interviews.

Analytic Approach. Using NVivo 12 software, a trained team of researchers coded interview transcripts. To organize data for analysis, we developed codes aligned with the care delivery requirements related to risk stratification and LCM. To code factors that practices described as barriers or

Table 1 Practice Characteristics of LCM Sample Selected for Indepth Study Versus All CPC+ Practices

\begin{tabular}{llll}
\hline \hline $\begin{array}{l}\text { Practice } \\
\text { characteristics }\end{array}$ & $\begin{array}{l}\text { LCM in-depth } \\
\text { study sample } \\
(\mathbf{\%})(\boldsymbol{N}=\mathbf{2 3})\end{array}$ & $\begin{array}{l}\text { All CPC+ } \\
(\mathbf{\%})(\boldsymbol{N}= \\
\mathbf{2 8 4 6})\end{array}$ & $\begin{array}{l}\text { Difference } \\
\mathbf{( \% )}\end{array}$ \\
\hline $\begin{array}{l}\text { Track 1 } \\
\text { Medicare Shared }\end{array}$ & 52 & 47 & 5 \\
$\begin{array}{l}\text { Savings Plan (SSP) } \\
\text { participant }\end{array}$ & 43 & 46 & 3 \\
$\begin{array}{l}\text { Independent } \\
\text { practice }\end{array}$ & 26 & 27 & 1 \\
$\begin{array}{l}\text { Practice size: } \\
\quad \text { Small-sized }\end{array}$ & 30 & 35 & 5 \\
practice \\
$\begin{array}{l}\text { Medium-sized } \\
\text { practice } \\
\text { Large-sized }\end{array}$ & 26 & 37 & 11 \\
practice & 43 & 28 & 15 \\
\hline
\end{tabular}

Source: Analysis of characteristics of $\mathrm{CPC}+$ practices included in the $L C M$ in-depth study and characteristics of $C P C+$ practices overall. Data were obtained from the CPC+ practice roster and the CMS application tracker and practice application file facilitators to risk stratification and LCM, we adapted the Consolidated Framework for Implementation Research. ${ }^{13}$ Researchers used NVivo queries of coded data to create analytic summaries and populated matrices with the analytic summaries to identify patterns across practices of barriers and facilitators relating to risk stratification and LCM. ${ }^{14}$

\section{RESULTS}

Our results include a quantitative assessment of the extent to which LCM reached high-risk patients in the second year of CPC+. Qualitative findings - including practices' approaches to risk stratification and LCM implementation, perceived benefits of LCM, and implementation challenges and facilitators-follow and provide context for the quantitative results. Results were similar for Track 1 and 2 practices, and practices fell roughly evenly between the two tracks [Table 1].

LCM Penetration. In CPC+'s second year, only a small proportion of all high-risk patients received LCM for their chronic conditions. In care delivery requirements data, practices reported placing a median of $2.4 \%$ of empaneled patients in the highest-risk tier. This is slightly lower than the 3 to $5 \%$ that CMS suggests $\mathrm{CPC}+$ practices with a typical population distribution designate as high risk. ${ }^{3}$ Furthermore, of the $2.4 \%$ in the highest-risk tier, only $30 \%$ (median) of patients received LCM [Fig. 1]. Practices placed a median of $10 \%$ of patients in the second-highest-risk tier; of these, $7 \%$ (median) received LCM. Only $1 \%$ or fewer of patients in the lower-risk tiers received LCM. In addition, CPC+ practices selected for indepth study noted that many patients who could benefit from LCM did not receive these services.

Use of Risk Stratification. In care delivery requirements data, most practices reported implementing a two-step risk stratification process, using a manual or an automated algorithm and clinical intuition to assign a risk score to each patient. Many practices selected for in-depth study, however, faced challenges implementing workflows to support two-step risk stratification. These challenges included a lack of buy-in among practitioners and staff regarding the value of risk scores to target certain patients, lack of confidence in how risk scores were assigned, and belief that risk scores did not help guide patients' care. In practices owned by health systems with centralized risk stratification, practitioners and staff perceived that risk scores were inaccurate or not updated to reflect patients' most current conditions. Practices also reported having insufficient or no EHR functionality to incorporate clinical intuition into risk stratification. For example, care team members could not update risk scores in their EHR to reflect a patient's most current clinical conditions and personal circumstances. 


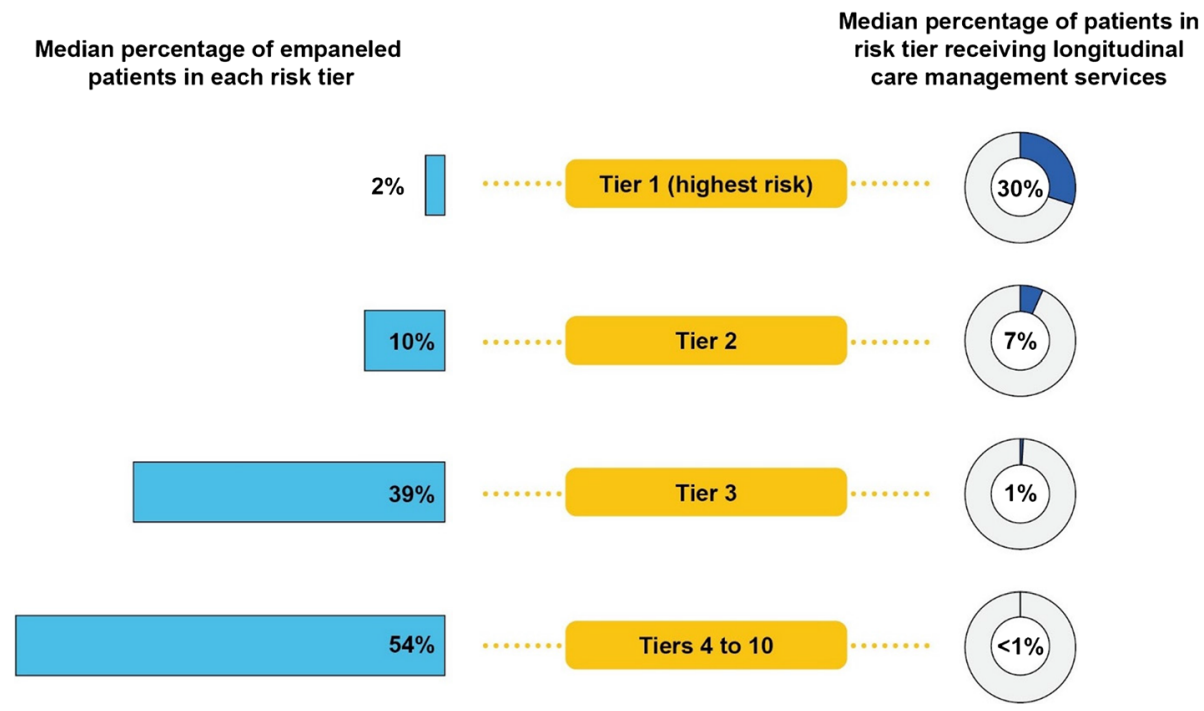

Figure $1 \mathrm{CPC}+$, year 2: median percentages of empaneled patients in each risk tier and median percentages of patients in each risk tier receiving LCM. Source: Mathematica's analysis of 2018 Q4 care delivery reporting data submitted by practices to CMS via the CPC+ practice portal. Note: For reporting purposes, CMS used the term "tier 1" to refer to the highest-risk tier, "tier 2" for the second highest, etc. Practices were only included in the risk tier calculations if they had at least one patient in a particular risk tier.

LCM Provision. Although CPC+ does not dictate who provides LCM, most practices selected for in-depth study reported having at least one part-time or full-time care manager (generally, a registered nurse or nurse practitioner) providing LCM to patients. In a few practices, the care manager was a social worker, medical assistant, or a care management team (such as a nurse-social worker team). Typically, care managers provided services in person and by phone. Topics of discussion included patients' conditions, recent changes in health status, medications, lifestyle and behavior choices, support systems, and behavioral health and social needs. Care managers communicated with primary care practitioners and staff (including behavioral health specialists and individuals providing comprehensive medication management services) to coordinate care for patients receiving LCM services. In some cases, care managers were embedded within practices; in other cases, (particularly in practices owned by health systems), care managers worked via phone from a centralized location at least part of the time and worked within practices at other times.

LCM Benefits. Many practices selected for in-depth study described the benefits of LCM for patients, including improved patient engagement and self-management of disease processes, which helps patients avoid the emergency department and hospital [Text Box 1]. For example, one care manager reported that when her practice piloted LCM, the first patient to receive LCM had six hospitalizations in the previous year. In the patient's first year receiving LCM, the patient had zero hospitalizations through improved self-management of their chronic conditions. In another practice, the practice manager reported continuous improvements in diabetic patients'
A1c levels since starting LCM and attributed these improvements to the care manager's intensive work with diabetic patients. A physician in a different practice appreciated the care manager's work with congestive heart failure patients. Frequent check-ins with these patients enabled the care manager to notify the practitioner when patients' weights rose; from the physician's perspective, this helped patients avoid the emergency department and hospital. Several practices also noted psychosocial benefits of LCM for patients, including feelings of gratitude, hope, and empowerment. Two practices specifically mentioned the benefits of LCM for elderly isolated patients, who especially appreciated the outreach and connection that LCM provides.

Text Box 1. Quote from primary care physician in a CPC+ practice

\begin{tabular}{l}
\hline "Our ultimate goal for doing the job is to give good patient care, which \\
in my mind is better quality, longer lives, and better quality lives... \\
We're making sure that medications are being taken...that weights are \\
being taken on a daily basis...that people are doing better with their \\
chronic disease, they're breathing better, their sugars are better...the \\
better patient outcome is really why we do [LCM]."
\end{tabular}

Several practices also reported the practice-level benefits of LCM. For example, care managers work through complex issues (medical and psychosocial) with patients, something practitioners might lack the time to do during office visits. A few practices reported that LCM resulted in care team members better understanding their roles and their teammates' roles, communicating better through the EHR, and being more prepared for appointments [Text Box 2]. Finally, two practices indicated that witnessing improvements in patients receiving LCM was gratifying to staff. 
Text Box 2. Quote from a primary care physician in a CPC+ practice

"When I open up a patient [chart], I can see immediately from their snapshot when [the care manager] has reached out, the day, time, and what was discussed...There's a transparency and accountability now that's new. That's one innovation I think is moving us towards better [LCM]."

LCM Challenges. Findings from practices selected for indepth study provided potential insight into why so few patients received LCM. Many noted that care managers had larger caseloads than they could manage [Text Box 3]. Practices also faced challenges hiring additional staff, particularly lack of funding for such hires and lack of available skilled care managers seeking employment. One physician noted that it is challenging to hire care managers with the knowledge, skills, and personality traits - such as patience, empathy, attention to detail, and a sense of "tough love" toward patients - that a good care manager needs. Even many practices that successfully hired care managers found integrating them effectively into care teams challenging. For example, a care manager in one practice noted that practitioners and other staff initially "didn't know what to do with [her]." Over time, clearly defining the care manager's duties and integrating her into care team meetings helped everyone understand her role and how to incorporate the care manager in the care of highrisk patients. In addition to staffing challenges, many practices selected for in-depth study had difficulty engaging patients in LCM because of what practices reported as psychosocial barriers, difficulty contacting patients, or patients' reluctance to change behaviors or lifestyle.

Text Box 3. Quote from a care manager in a $\mathrm{CPC}+$ practice

"It's a lot. Some days I'm just barely swimming above water... The biggest problem is that I'm spread too thin."

Another challenge to implementing LCM was developing and using care plans. $\mathrm{CPC}+$ required practitioners and care managers in more advanced (Track 2) $\mathrm{CPC}+$ practices to develop and maintain care plans for patients receiving LCM. CMS defines a care plan as a mutually agreed upon and documented plan of care based on the patient's goals, needs, and self-management activities accessible to all team members caring for the patient. Care plans are structured, standardized, and commonly include treatment goals and interventions identified by the care team, the patient's overall health goals, advance directives, key contact information for practices, and actions that the patient and the care team plan to take. Several care managers in the in-depth study described using such care plans, but several others conflated care plans with other forms of clinical documentation, such as after-visit summaries and progress or encounter notes. Nearly all practices selected for in-depth study reported challenges using EHRs to create, access, and update care plans. In particular, care managers described challenges related to creating custom care plan templates after discovering that their EHR-provided care plans were disease specific and not whole-person oriented. Others reported that they could store care plans in their EHRs, but they could not create or update care plans in them; care managers had to upload care plan files to the patient record every time they updated a patient's care plan.

LCM Facilitators. Practices selected for in-depth study noted some facilitators to implementation. Many practices perceived the benefits of systematically identifying and prioritizing patients for LCM based on their care needs and their health care utilization [Text Box 4]. Several practices said that having care managers physically located in the practice enabled them to join care team huddles and helped other forms of communication (such as care managers touching base with practitioners after meeting with patients) throughout the day [Text Box 5]. Practices also reported that having care managers embedded in the practice facilitated warm hand-offs of patients from practitioners to care managers and helped patients establish trust with the care manager. Several practices described aspects of their health information technology systems that made providing LCM easier, including care managers' use of EHRs to review patients' histories and identify gaps in care, build registries to track outreach to patients and patients' progress toward health goals, and communicate with other members of the care team. Several practices using whole-person-centered care plans reported that they helped care managers quickly refresh their deep understanding of patients' conditions, reference and track patients' progress toward goals, tailor patient follow-ups, and share information with other members of the care team.

Text Box 4. Quote from a primary care physician in a CPC+ practice

"[Risk stratification] really does allow us...to put the right resource at the right population of patients, impact their quality of life, impact their adherence, and keep them out of the hospital."

Text Box 5. Quote from a primary care physician in a CPC+ practice

"[Having an onsite care manager is] a game changer. It can take some of the burden off of the physician for phone calls and follow-up...I think it's huge in our ability to care for patients [and] decrease avoidable ER visits and inpatient admissions."

\section{DISCUSSION}

Even with substantial CPC+ financial and other supports to practices for risk stratification and LCM, less than a third of patients identified as being in the highest-risk tier received 
LCM. CMS guidance to CPC+ practices indicated that typically, $3-5 \%$ of a population would be classified as high risk, with no more than $10 \%$ of the total patient population receiving LCM. However, in the second year of CPC+, only $30 \%$ (median) of the $2.4 \%$ of patients (median) identified as high risk received LCM. In addition, only $7 \%$ (median) of the $10 \%$ (median) of patients in the second-highest-risk tier received LCM. While we cannot determine the exact percentage of patients in CPC+ who received LCM in 2018, it appears that practices provided far less than $10 \%$ of their patient population with these services. In addition, CPC+ practices selected for in-depth interviews noted that many patients who could benefit from LCM did not receive it.

$\mathrm{CPC}+$ practices acknowledge many benefits of providing LCM to high-risk patients, but successfully implementing LCM depends on many interacting factors, including perceptions among practitioners and staff that risk stratification adds value, staff availability and expertise to provide LCM, EHR functionalities to support risk stratification and care plan development and use, and care teams' confidence in the accuracy of their risk stratification process to identify high-risk patients for LCM. For many practices, the challenges of implementing LCM hinder the provision of these services to many patients who could benefit from them.

A recent systematic review underscored risk stratification's foundational importance as a precursor to LCM, finding that accurate risk stratification (that is, identifying patients for whom there is greatest opportunity to benefit from LCM) was a key factor in producing positive outcomes of LCM, such as better health, functional status, self-management; and lower emergency department visits, hospital admissions (and length of stay), and emergency department and inpatient costs. ${ }^{15}$ Our study's findings, however, suggest that some practitioners and staff lack trust in risk stratification processes and report that the process is not well supported by EHR functionalities. In addition, some practitioners and staff do not believe risk stratification has value or can help them better serve patients. Primary care practices juggling the competing demands of delivering high-quality patient care (and in many cases, also participating in transformation efforts such as $\mathrm{CPC}+$ ) might require intensive support to effectively and efficiently implement risk stratification. Stronger evidence of the connection between effective risk stratification and desirable patient outcomes could help achieve buy-in from practitioners and staff who question the necessity and value of this process.

Another key factor shaping positive outcomes in LCM identified in the recent systematic review is providing "highintensity intervention" (that is, small caseloads for care managers, initial in-person patient assessments, multidisciplinary team meetings to discuss patients, and development of care plans that include the perspectives of multiple types of providers). ${ }^{15}$ Although this could be the ideal way to deliver LCM, our findings suggest that practices might struggle to achieve this because they lack qualified staff to deliver LCM.
For practices not participating in $\mathrm{CPC}+$, which provides financial and other supports to implement LCM, the problem of adequate staffing to provide enhanced care management could be even more pronounced. For example, despite recommendations to expand the employment of nurses and other health professionals in primary care and care management, ${ }^{16,}{ }^{17}$ nearly $40 \%$ of practices that participated in the 2018 national cross-sectional Survey of Primary Care and Geriatric Clinicians employed neither a social worker nor a registered nurse $(N=410$ clinicians in 363 practices that provide care to older adults). ${ }^{18}$

Care management staffing challenges in primary care might stem from many different sources. As practices in our in-depth study noted, despite an influx of CPC+ payments, funding limitations prevented hiring of more care managers. It is also difficult to hire effective care managers who have the skills and the personality traits that make them ideal for the job. Care management is part of nursing school curricula but often focuses on acute care settings and transitions to communitybased care rather than providing LCM in primary care settings. ${ }^{18}$ Even when primary care practices hire a qualified, experienced care manager, they could face challenges integrating the care manager into care teams and workflows. Primary care practices seeking to integrate care managers into their teams might benefit from resources and training on topics such as effectively communicating within the team, delegating particular tasks from practitioners to care managers, practicing warm hand-offs of patients to care managers, and clearly delineating roles and responsibilities of the care manager and other care team members. ${ }^{19,} 20$

While our mixed-methods approach allows us to triangulate quantitative and qualitative data on LCM uptake, our study has limitations. Practices self-reported data on the number of patients in each risk tier and, among them, the number receiving LCM. Because of the relatively low rates of LCM uptake reported, however, we suspect that self-reporting bias (social desirability bias to overreport) is not a problem. In addition, practices selected for in-depth study represent a small proportion of CPC+ practices, but the characteristics of this sample reflect the characteristics of $\mathrm{CPC}+$ practices overall in terms of track; participation in the Medicare Shared Savings Program; whether they were independent or owned by a system, hospital, or large group practice; and size (as measured by number of billing practitioners at the practice site). Finally, the practices and patients in $\mathrm{CPC}+$ might not generalize to practices nationwide or to other patient-centered medical home models.

In sum, CPC+ provides valuable lessons about implementing LCM. Despite CPC+ funding and other supports, the proportion of high-risk patients receiving LCM in participating practices remains low, and barriers to providing these services persist. To expand the reach of LCM, practices would benefit from additional care managers, training for staff to overcome barriers to engage patients in care management, and increased practitioner and staff buy-in to the concept and value of risk stratification. Further investigation into whether 
and how current volume-based payment incentives contribute to these barriers is necessary.

Acknowledgments: Deborah N. Peikes, Ph.D., M.P.A., a senior fellow at Mathematica and Project Director and Co-Principal Investigator of the evaluation of $\mathrm{CPC}+$, critically reviewed this manuscript. Rumin Sarwar, M.S., a researcher at Mathematica, contributed analysis related to care plans.

Corresponding Author: Tricia Collins Higgins, Ph.D., M.P.H.; Mathematica, Washington, DC, USA (e-mail: thiggins@mathematicampr.com).

Funding The work was funded by the Department of Health and Human Services, Centers for Medicare \& Medicaid Services under contract HHSM-500-2014-00034I/HHSM-500-T0010.

\section{Compliance with Ethical Standards:}

Conflict of Interest: The authors declare that they do not have a conflict of interest.

Disclaimer: The contents of this manuscript are solely the responsibility of the authors and do not necessarily represent the official views of the U.S. Department of Health and Human Services or any of its agencies.

\section{REFERENCES}

1. Peikes D, Anglin G, Harrington $\mathbf{M}$, et al. Independent evaluation of Comprehensive Primary Care Plus (CPC+): first annual report. Princeton (NJ): Mathematica; 2019 April. Contract No: HHSM-500-2014-000341/ HHSM-500-T0010. Sponsored by the U.S. Department of Health and Human Services, Center for Medicare \& Medicaid Innovation.

2. Anglin G, Peikes D, Petersen D, et al. Independent evaluation of Comprehensive Primary Care Plus (CPC+): second annual report. Princeton (NJ): Mathematica; 2020 July. Contract No: HHSM-5002014-00341/HHSM-500-T0010. Sponsored by the U.S. Department of Health and Human Services, Center for Medicare \& Medicaid Innovation.

3. Centers for Medicare \& Medicaid Services. 2018 CPC+ implementation guide: guiding principles and reporting. Washington, DC: CMS, January 30, 2018.

4. Berry-Millet R, Bodenheimer TS. Care management of patients with complex health care needs. Synth Proj Synth Rep. 2009; 19:52372.

5. Wilson C, O'Malley AS, Bozzolo C, McCall N, Ma S. Patient experiences with chronic care management services and fees: a qualitative study. J Gen Intern Med. 2019;34(2):250-255.
6. Ganguli I, Orav EJ, Weil E, Ferris TG, Vogeli C. What do high-risk patients value? Perspectives on a care management program. J Gen Intern Med. 2017;33(1):26-33.

7. Hsu J, Price M, Vogeli C, et al. Bending the spending curve by altering care delivery patterns: the role of care management within a Pioneer ACO. Health Aff. 2017;36(5):876-884.

8. McWilliams JM, Chernew ME, Landon BE. Medicare ACO program savings not tied to preventable hospitalizations or concentrated among high-risk patients. Health Aff. 2017;36(12):2085-2093.

9. Garcia ME, Uratsu CS, Sandoval-Perry J, Grant RW. Which complex patients should be referred for intensive care management? A mixed methods analysis. J Gen Intern Med. 2019;33(9):1454-1460.

10. Wagner J, Hall JD, Ross RL, et al. Implementing risk stratification in primary care: challenges and strategies. J Am Board Fam Med. 2019;32:585-595.

11. Hong CS, Abrams MK, Ferris TG. Toward increased adoption of complex care management. N Engl J Med. 2014;371(6):491-493.

12. Centers for Medicare \& Medicaid Services. Comprehensive Primary Care Plus. Available at: https://innovation.cms.gov/innovation-models/comprehensive-primary-care-plus. Accessed June 3, 2020.

13. Damschroder LJ, Aron DC, Keith RE, Kirsh SR, Alexander JA, Lowery JC. Fostering implementation of health services research findings into practice: a consolidated framework for advancing implementation science. Implement Sci. 2009;4(50).

14. Miles MB, Huberman AM, Saldaña J. Qualitative data analysis: a methods sourcebook. 3rd ed. Thousand Oaks: Sage Publications; 2014.

15. Hudon C, Chouinard $\mathbf{M}$, Pluye $\mathbf{P}$, et al. Characteristics of case management in primary care associated with positive outcomes for frequent users of health care: a systematic review. Ann Fam Med. 2019; 17(5):448-458.

16. Blumenthal D, Chernof B, Fulmer T, Lumpkin J, Selberg J. Caring for high-need, high-cost patients -an urgent priority. N Engl J Med. 2016;375(10):909-911.

17. Bodenheimer T, Bauer L. Rethinking the primary care workforce - an expanded role for nurses. N Engl J Med. 2016;375(11):1015-1017.

18. Donelan K, Chang Y, Berrett-Abebe J, Spetz J, Auerback DI, Norman L, Buerhaus PI. Care management for older adults: the roles of nurses, social workers, and physicians. Health Aff. 2019;38(6):941-949.

19. O'Malley AS, Gourevitch R, Draper K, Bond A, Tirodkar MA. Overcoming challenges to teamwork in patient-centered medical homes: a qualitative study. J Gen Intern Med. 2015;30(2):183-192.

20. Johnson C, Houy M. Role of the nurse care manager in a patientcentered medical home: lessons learned from the Massachusetts PatientCentered Medical Home Initiative. CMSA Today. 2013; Issue 2. Available at: https://www.nxtbook.com/nxtbooks/naylor/CMSQ0412/index. php?startid=8\#/p/8. Accessed October 12, 2020.

Publisher's Note Springer Nature remains neutral with regard to jurisdictional claims in published maps and institutional affiliations. 